\title{
Visualization of Similar Patients in a Clinical Decision Support System for Rare Diseases - A Focus Group Study
}

\author{
Jannik SCHAAF ${ }^{\mathrm{a}, 1}$, Martin SEDLMAYR ${ }^{\mathrm{b}}$, Hans-Ulrich PROKOSCH ${ }^{\mathrm{c}}$, \\ Niels TEGTBAUER ${ }^{\mathrm{a}}$, Dennis KADIOGLU ${ }^{\mathrm{a}}$, Johanna SCHAEFER ${ }^{\mathrm{a}}$, \\ Martin BOEKER ${ }^{\mathrm{d}}$ and Holger STORF ${ }^{\mathrm{a}}$ \\ ${ }^{a}$ Medical Informatics Group, University Hospital Frankfurt, Frankfurt, Germany \\ ${ }^{b}$ Institute for Medical Informatics and Biometry, Carl Gustav Carus Faculty of \\ Medicine Technical University of Dresden, Dresden, Germany \\ ${ }^{c}$ Chair of Medical Informatics, Department of Medical Informatics, Biometrics and \\ Epidemiology, Friedrich-Alexander University Erlangen-Nürnberg, Erlangen, \\ Germany \\ ${ }^{d}$ Institute of Medical Biometry and Statistics, Medical Faculty and Medical Centre- \\ University of Freiburg, Freiburg, Germany
}

\begin{abstract}
The diagnosis of patients with rare diseases is often delayed. A Clinical Decision Support System using similarity analysis of patient-based data may have the potential to support the diagnosis of patients with rare diseases. This qualitative study has the objective to investigate how the result of a patient similarity analysis should be presented to a physician to enable diagnosis support. We conducted a focus group with physicians practicing in rare diseases as well as medical informatics researchers. To prepare the focus group, a literature search was performed to check the current state of research regarding visualization of similar patients. We then created software-mockups for the presentation of these visualization methods for the discussion within the focus group. Two persons took independently field notes for data collection of the focus group. A questionnaire was distributed to the participants to rate the visualization methods. The results show that four visualization methods are promising for the visualization of similar patients: "Patient on demand table", "Criteria selection", "Time-Series chart" and "Patient timeline. "Patient on demand table" shows a direct comparison of patient characteristics, whereas "Criteria selection" allows the selection of different patient criteria to get deeper insights into the data. The "Time-Series chart" shows the time course of clinical parameters (e.g. blood pressure) whereas a "Patient timeline" indicates which time events exist for a patient (e.g. several symptoms on different dates). In the future, we will develop a software-prototype of the Clinical Decision Support System to include the visualization methods and evaluate the clinical usage.
\end{abstract}

Keywords. Rare Diseases, Clinical Decision Support, Requirements analysis

${ }^{1}$ Corresponding Author, Jannik Schaaf, Medical Informatics Group, University Hospital Frankfurt, Theodor-Stern-Kai 7, 60590 Frankfurt am Main, Germany; E-mail: Jannik.schaaf@kgu.de. 


\section{Introduction}

In the EU a disease is declared as rare if it affects less than 1 of 2000 people. The diagnosis of rare diseases (RDs) is often delayed [1]. Since over 7000 RDs exist, a physician cannot be aware of all existing RDs. In addition, experts of RDs are often distributed regionally and the availability of data for research is low [2]. Hence, it is useful to connect data of RDs and make it available for research and care. The linkage of datasets inside large research networks provides a great opportunity to combine medical knowledge of different institutions. The "Medical Informatics Funding Scheme", created by the German Ministry of Education and Research (BMBF), establishes four consortia to set up data integration centers (DICs) at each university hospital [3]. The goal is to bundle data for research and care. One of these consortia is MIRACUM (Medical Informatics in Research and Care in University Medicine) which includes 10 university hospitals [3]. To demonstrate the use of data sharing, MIRACUM established a use-case about the conception, implementation and evaluation of a Clinical Decision Support System (CDSS) for RDs. Hunt et al. define a CDSS as a system that supports clinical decision making and compares clinical features of a patient with a knowledge base and makes these results available to physicians [4]. The planned CDSS shall support the diagnosis of an undiagnosed patient by comparing the undiagnosed patient with diagnosed patients from the DICs. The results of the similarity analysis should be visualized to the physicians [5].

To develop a meaningful and successful CDSS in MIRACUM, it is essential to determine how similar patients should be visualized to the physicians. Therefore, the objective of this study is to determine how results of the similarity analysis of patients with RDs should be presented to the user to enable diagnosis support in a CDSS.

\section{Methods}

We conducted a focus group to investigate the visualization of similar patients for a CDSS for RDs. The aim of the focus group is to have an intensive discussion about a specific topic in which the subjective experiences of participants within a group are important [6]. The study was performed and reported in accordance with the SRQR Guideline (Standards for Reporting Qualitative Research) [7]. We considered 21 out of 22 items of SRQR. In this section, we describe the method steps that were performed for the focus group. We prepared the focus group with a literature search and created mockups based on the results of the search. We then recruited the study participants and conducted the focus group. Finally, the results of the focus group were analyzed.

\subsection{Literature search}

The aim of the literature search was to identify visualization methods that can be used for the representation of similar patient cases. Therefore, we searched PubMed between January 15th, 2009 and January 15th, 2019. The search was conducted at January 16, 2019. The search was based on keywords from the two search groups "A" and "B", including MeSH Terms and non-MeSH Terms. Group A included the term "Patient similarity". Group B included the terms "Visualization", "Visual analytics", "Knowledge discovery and exploration", "Data Display (MeSH)" and "Decision Support Systems, Clinical (MeSH)". The search groups were linked by a logical "AND". All search results 
were limited to English articles. We included original peer reviewed journals and conference papers. All other publication types were excluded. In addition, we only considered publications that address the visualization of similar patients.

Software mockups were used to allow a discussion about the visualization methods in the focus group. Mockups are drafts (e.g. in the form of images) of a software interface. The mockups include visualization methods of the literature search. For the discussion about the mockups, an interview-guide was prepared which was used to structure and deepen the discussion [8]. The interview-guide was developed as advised by Mayring as a basis for the discussion of each visualization method [9]. The interview-guide contains four questions: (1) "What do you think about the visualization method?" (2) "How would you use this information to enable a diagnosis for the patient?" (3) "Do you have any suggestions for improvement?" (4) "How do you rate the visualization method? What are advantages or disadvantages?”

\subsection{Recruitment and conduction of the focus group}

A purposeful sampling was used to recruit participants used [9]. We contacted physicians who work with RDs patients in the MIRACUM consortia. Furthermore, we contacted non-physicians in the MIRACUM consortia with research-experience in medical informatics. We sent an email to eight experts of RDs in January 2019, representing eight specialized centers for RDs in MIRACUM. We further sent an email via a distribution list of MIRACUM to recruit the non-physicians.

The focus group took place in March 2019 in a seminar room. The duration was 120 minutes and the language was German. However, there is no standardized procedure for data collection and analysis of focus groups. It depends on the research question and the possible timeframe for the study. Therefore, we decided to make field-notes of the statements within the focus group and did not record the focus group with an audio device. Field notes were taken by two persons (NT and JOS) to reduce bias and increase accuracy [10]. Personal identifying data of study participants were not included in the field notes. The focus group was moderated by JAS, whose researcher characteristics are the following: "gender: Male", "experience: 3 years research experience in medical informatics, main focus in RD projects", "degree: M.Sc. in Medical Informatics", "occupation: Research assistant".

\subsection{Data analysis of the focus group}

We used a deductive approach with a qualitative content analysis as advised by Mayring and built categories before data analysis to extract text passages of the field notes [9]. An interview-guide was created based on four questions, as stated in section 2.1. If the field notes contained a statement in terms of a visualization method, these text passages were extracted. Therefore, a deductive category was created for each visualization method. Text passages were extracted from the field notes if they contained a statement to a visualization method. Whenever a text passage could not be clearly assigned to a category, the text passage was discussed and modified where required by NT, JAS and JOS. Furthermore, a questionnaire was used to evaluate the focus group, which was distributed to the participants at the end of the discussion. The questionnaire included ten items to rate the visualization method of the mockups with an ordinal scale from five (very important) to one (not important). The scale was derived from Schulz et al. to rate visualization elements of an information element [10]. For data analysis the visualization 
methods were compared with the field notes of the focus group. The questionnaire also provided the options to write a comment about the visualization methods. These comments were also assigned to the deductive categories. Quotations of field notes and comments were translated from German into English for publication. Saturation of this study was reached when all categories were adequately represented in the data [11].

\section{Results}

\subsection{Selection of stimuli for the focus group}

The literature research identified 184 results. Five publications were identified as relevant [12-15]. During a discussion, all authors came to an agreement that Radar Plots and Neighborhood Related Graphs could be excluded, since they were irrelevant for the focus group [16]. Neighborhood Related Graphs show the proximity or distance between similar patients, but do not provide a quantitative representation of the similarities between patients. On the other hand, Radar plots require at least three categories of data in order to display the chart, otherwise two existing lines lie on top of each other and no further connection can be identified. Hence, the chart does not offer the possibility to compare two data categories directly and was therefore not used for the focus group.

In the next step, mockups with a maximum of five visualization methods were created. The mockups were displayed as images during the presentation in the focus group. Mockup 1 shows four different sections with one visualization method for each section. The section "Overview of similar patients" shows a "Patient similarity plot". It shows similar patients in regard to their similarity plotted on a scale from 0 (not similar) to 1 (most similar). According to Stubbs et al., "Criteria selection" can be used to calculate the similarities of patients to certain criteria [13]. A physician may be interested in displaying similar patients only based on symptoms. The section "Similar criteria" displays the distribution of the selected similarity criteria as a "Ranking glyph" [14]. Different rankings display how strongly a criteria affects the overall similarity. The section "Comparison of individual parameters" shows the progression of certain medical parameters between of two patients in a "Time-Series chart" [13].

Mockup 2 shows a "Treemap" in the section "Overview of similar patients" containing rectangles sorted by size. The size of the rectangle indicates the similarity of rectangles sorted by size. The size of the rectangle indicates the similarity of patients [15]. The section "Criteria selection" is the same as in Mockup 1. The section "Patienttimeline" shows the history of a patient with the option to click on further details, whereas "Distribution of specific parameters" shows the distribution of data that originates from certain criteria, such as symptoms or diagnoses, via "Pie-chart". 

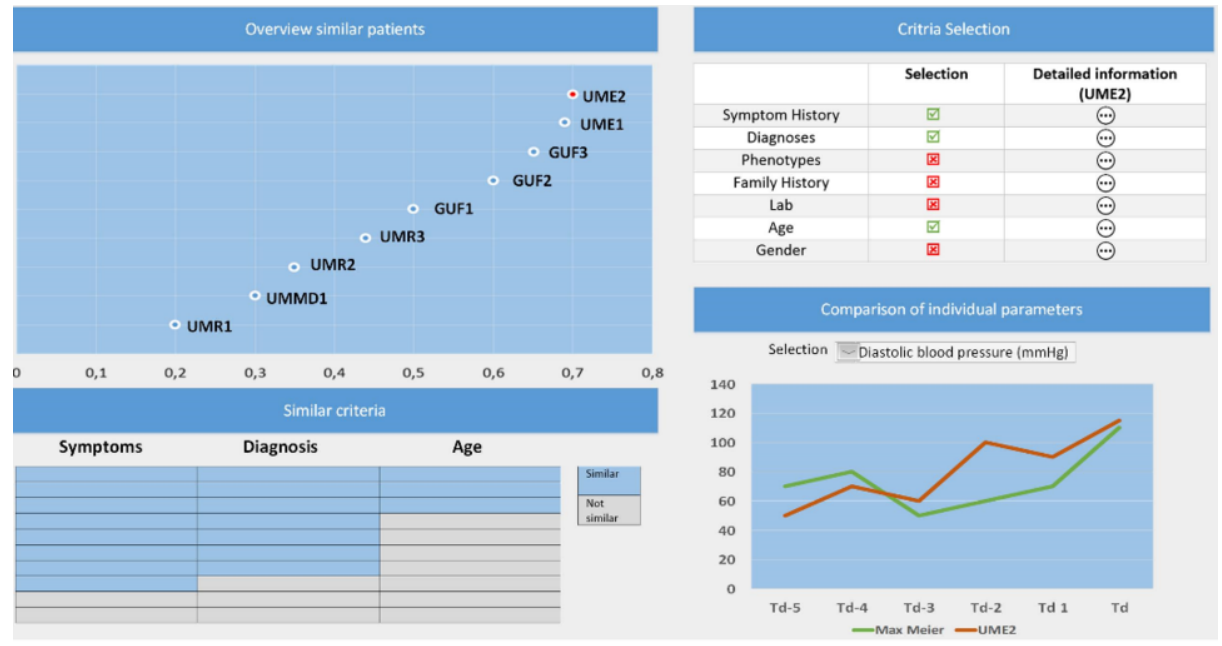

Figure 1. Mockup 1 for the focus group
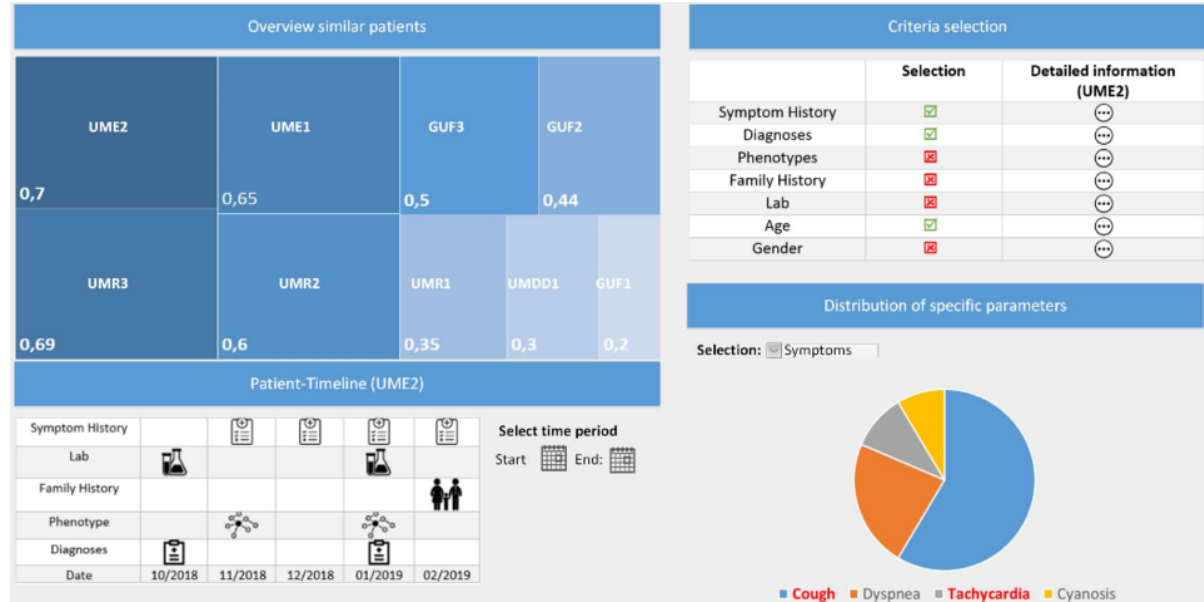

Selection: Symptoms

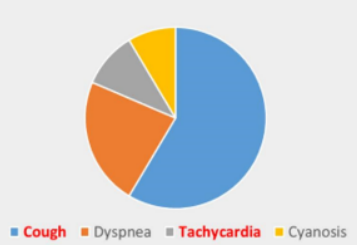

Figure 2. Mockup 2 for the focus group

In Mockup 3, "Overview of similar patients" and "Criteria selection" are the same as in Mockup 1. The section "Distribution of all patients" shows the distribution of patients on the basis of a "Sunburst plot" [14]. It displays the distribution of diagnoses and their relationships to other diagnoses across all patients. The section "Correlations" shows a heatmap with correlations between different types of data $[14,15]$. The last visualization method shows the comparison of individual patient properties in a "Patient on demand table". 


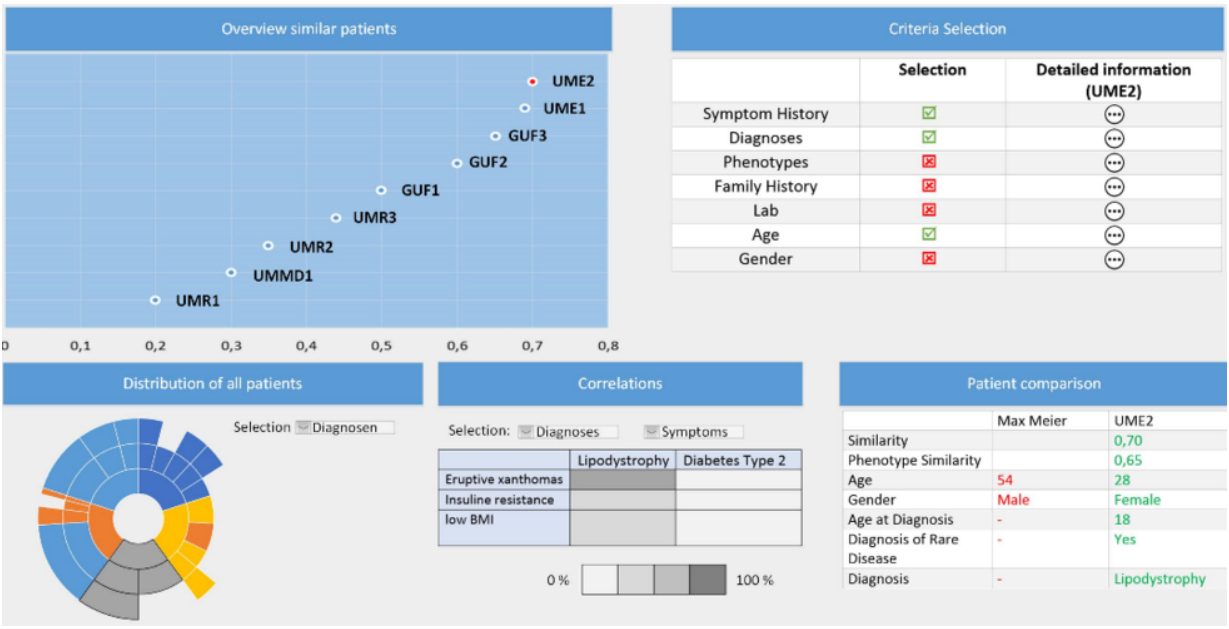

Figure 3. Mockup 3 for the focus group

\subsection{Results of the focus group}

Six out of eight experts for RDs responded to our invitation. Two of the invited experts could not be reached by phone or email. Furthermore, 11 non-physicians registered for the focus group. In total, 17 participants took part in the focus group. The score of one visualization method per participant was determined, summed up and an average was calculated (shown in Table 1).

Table 1. Results of the questionnaire for all visualization methods of the mockups

\begin{tabular}{llll}
\hline $\begin{array}{l}\text { Visualization method / } \\
\text { Category }\end{array}$ & Visualization methods & Total points & Average \\
\hline 1 & Patient similarity plot & 49 & 3.06 \\
\hline 2 & Criteria selection & 71 & 4.17 \\
\hline 3 & Ranking glyph & 51 & 3.18 \\
\hline 4 & Time-Series chart & 71 & 4.17 \\
\hline 5 & Treemap & 44 & 2.75 \\
\hline 6 & Patient timeline & 70 & 4.11 \\
\hline 7 & Pie-chart & 44 & 2.93 \\
\hline 8 & Sunburst plot & 43 & 2.68 \\
\hline 9 & Heatmap & 58 & 3.44 \\
\hline 10 & Patient on demand table & 74 & 4.35 \\
\hline
\end{tabular}

\subsubsection{Results of the categories and comparison with the questionnaire}

\section{Category 1: Patient similarity plot}

The participants indicated for the category "Patient similarity plot" that this graph is important to determine the similarity. One participant stated that it is important to offer several patients for comparison. Other participants indicated that a tabular presentation would be clearer for an overview of similar patients. However, the "Patient similarity plot" does not lead to the best results in the questionnaire.

\section{Category 2: Criteria selection}

Regarding the category "Criteria selection", the participants indicated that a weighting of the selection criteria should be possible. This is consistent with the ranking in the 
questionnaire. One participant said: "How are the weightings set? Assuming that this is an $\mathrm{RD}$, is it useful to weight certain parameters, e.g. is it conspicuous if two symptoms occur together?"

\section{Category 3: Ranking glyph and Category 4: Time-Series chart}

The "Ranking glyph" was rated negatively, both in the questionnaire and in the statements of the participants. The "Time-series chart" was evaluated positively in the discussion and in the comments of the questionnaire. It also achieved a high score in the questionnaire.

\section{Category 5: Treemap}

According to the questionnaire, category 5 is not considered as useful visualization method and achieves a score of 2.75. This is consistent with numerous comments and the field notes, e.g.: "In the visualization, it is not easy to see where the similarity exists".

\section{Category 6: Patient timeline}

Category 6 is the third most important visualization instrument according to the questionnaire (4.11 points). In the field notes, the temporal arrangement and rhythm in the visualization is considered important.

\section{Category 7: Pie-chart, Category 8: Sunburst plot and Category 9: Heatmap}

"Pie-chart", "Sunburst plot" and "Heatmap" were rated, e.g. as "too complicated", "not recognizable" and "not relevant". This is consistent with the questionnaire ( $<3.5$ points).

\section{Category 10: Patient on demand table}

This category scores the best in the questionnaire ( 4.35 points). In a commentary the visualization method is given as simple and clear.

\section{Discussion}

The results show that statements of participants in the focus group were similar to the results of the questionnaire. The top four rated visualization methods are "Patient on demand table", "Criteria selection", "Time-Series chart" and "Patient timeline". However, Time-Series charts and Patient timelines are used in other medical applications $[17,18]$. We could not find any other publications with regard to "Patient on demand table" and "Criteria selection".

Using software-mockups as a "low-fidelity prototype" are a first step to show ideas to the future users in a previous stage of the development of a CDSS. Since low-fidelity prototypes do not allow user interactions, a high-fidelity prototype, with a real software functionality, might have other impacts on the results. High fidelity prototypes are assumed to deliver more effective data of the human interaction with a software [19]. Therefore we will develop a software prototype and evaluate in a further study which of the visualization methods are practicable. One possibility could be a so-called "Thinking Aloud Test", where the users test the system and communicate their thoughts and suggestions [20].

\section{Limitations}

The study has the following limitations. It was only possible to include participants of the MIRACUM consortium. Therefore, the results are limited to MIRACUM and Germany. Moreover, the proportion of physician participants was significantly lower, 
what could have an impact on the results. We did not record the focus group and therefore no verbatim transcript is available. However, we minimized possible bias across the study by taking field notes by two persons independently and by implementing a high methodological standard with SRQR.

\section{Conclusion}

In this work, we could show that relevant methods for the visualization of RDs are available. Looking ahead, we will develop a software prototype for our CDSS including these visualization methods. Only a further user-centered evaluation will show, whether the selected visualization methods are effectively suitable for the representation of similar patients in RDs.

\section{Acknowledgments}

MIRACUM is funded by the German Federal Ministry of Education and Research (BMBF) within the "Medical Informatics Funding Scheme" (FKZ 01ZZ1801A, 01ZZ1801B, 01ZZ1801C, 01ZZ1801L).

\section{References}

[1] Knight A, Senior T, The common problem of rare disease in general practice, Med J Aust. 185 (2006) 82-83.

[2] Storf H, Schaaf J, Kadioglu D, Goebel J, Wagner T.O.F., Ueckert F, Registries for rare diseases: OSSE - An open-source framework for technical implementation, Bundesgesundheitsblatt Gesundheitsforschung Gesundheitsschutz. 60 (2017), 523-531.

[3] Prokosch H, Acker T, Bernarding J, Binder H, Boeker M, Boerries M et al., MIRACUM: Medical Informatics in Research and Care in University Medicine - A Large Data Sharing Network to Enhance Translational Research and Medical Care, Methods Inf Med. 57 (2018), 82-91.

[4] Hunt D, Haynes R, Hanna S, Smith K, Effects of computer-based clinical decision support systems on physician performance and patient outcomes: a systematic review, JAMA. 280 (1998), 1339-1346.

[5] Schaaf J, Boeker M, Haverkamp C, Hermann T, Kadioglu D, Prokosch H et al., Finding the Needle in the Hay Stack: An Open Architecture to Support Diagnosis of Undiagnosed Patients, Stud Health Technol Inform. (2019), 1580-1581.

[6] Krueger R, Focus groups: a practical guide for applied research, 2nd ed., Sage Publications, Thousands Oaks (CA), 1994.

[7] O'Brien BC, Harris I, Beckman T, Reed D, Cook D, Standards for reporting qualitative research: a synthesis of recommendations, Acad Med. 89 (2014).

[8] Tausch A, Menold N, Methodische Aspekte der Durchführung von Fokusgruppen in der Gesundheitsforschung - Welche Anforderungen ergeben sich aufgrund der besonderen Zielgruppen und Fragestellungen?, GESIS (2015).

[9] Mayring P, Qualitative Inhaltsanalyse. Grundlagen und Techniken, 12th ed. Beltz Verlag, Weinheim Basel, 2010.

[10] Schulz M, Mack B, Renn O, Fokusgruppen in der empirischen Sozialwissenschaft - Von der Konzeption bis zur Auswertung, Springer VS, Wiesbaden, 2012.

[11] Saunders B, Sim J, Kingstone T, Baker S, Waterfield J, Bartlam B et al., Saturation in qualitative research: exploring its conceptualization and operationalization, Qual. Quant. 52 (2018), 1893-1907.

[12] Gotz D, Sun J, Cao N, Ebadollahi S, Visual cluster analysis in support of clinical decision intelligence, AMIA Annu. Symp. Proc. (2011), 481-490.

[13] Stubbs B, Kale D.C., Das A, Sim TwentyFive: an interactive visualization system for data-driven decision support, AMIA Annu. Symp. P. (2012), 891-900. 
[14] Fan D, Plaisaint C, Spring N, Shneidermann B, Visual Interfaces for Recommendation Systems: Finding Similar Dissimilar Peers Sunburst Plots", (2018). http://frankdu.org/papers/du2018tist.pdf (accessed March 26, 2020).

[15] Tsymbal A, Huber M, Zillner S, Hauer T, Zhou S.K., Visualizing Patient Similarity in Clinical Decision Support. WA 2007 - Lernen - Wissen - Adaptivitat - Learning, Knowledge, and Adaptivity, Workshop Proceedings. (2007), 304-311.

[16] Saary M.J., Radar plots: a useful way for presenting multivariate health care data, J. Clin. Epidemiol. 61 (2008), 311-317.

[17] Ledesma A, Bidargaddi N, Strobel J, Schrader G, Nieminen H, Korhonen I et al., Health timeline: an insight-based study of a timeline visualization of clinical data, BMC Med. Inform. Decis. Mak. 19 (2019), 170.

[18] Bui A. A. T., Aberle D.R., Kangarloo H, TimeLine: Visualizing Integrated Patient Records, IEEE Trans. Inf. Technol. Biomed. 11 (2007), 462-473.

[19] Walker M, Takayama L, Landay J, Leila T, High-Fidelity or Low-Fidelity, Paper or Computer Choosing Attributes When Testing Web Prototypes, Proc. Hum. Factors Ergon. Soc. Annu. Meet. 46 (2002).

[20] Boren T, Ramey J, Thinking Aloud: Reconciling Theory and Practice, IEEE transactions on professional communication. $\mathbf{4 3}$ (2000). 